\title{
Characterization and stability measurement of deployed multicore fibers for quantum
} applications

Bacco, Davide ; Biagi, Nicola ; Vagniluca, llaria ; Hayashi, Tetsuya ; Mecozzi, Antonio ; Antonelli, Cristian ; Oxenløwe, Leif K.; ZavattaZavatta, Alessandro

Published in:

Photonics Research

Link to article, DOI:

$10.1364 / P R J .425890$

Publication date:

2021

Document Version

Publisher's PDF, also known as Version of record

Link back to DTU Orbit

Citation (APA):

Bacco, D., Biagi, N., Vagniluca, I., Hayashi, T., Mecozzi, A., Antonelli, C., Oxenløwe, L. K., \& ZavattaZavatta, A. (2021). Characterization and stability measurement of deployed multicore fibers for quantum applications. Photonics Research, 9(10), 1992-1997. https://doi.org/10.1364/PRJ.425890

\section{General rights}

Copyright and moral rights for the publications made accessible in the public portal are retained by the authors and/or other copyright owners and it is a condition of accessing publications that users recognise and abide by the legal requirements associated with these rights.

- Users may download and print one copy of any publication from the public portal for the purpose of private study or research.

- You may not further distribute the material or use it for any profit-making activity or commercial gain

- You may freely distribute the URL identifying the publication in the public portal 


\title{
PHOTONICS Research
}

\section{Characterization and stability measurement of deployed multicore fibers for quantum applications}

\author{
Davide Bacco, ${ }^{1, *}$ (1) Nicola Biagi, ${ }^{2,3}$ (1) Ilaria Vagniluca, ${ }^{2,4}$ Tetsuya Hayashi, ${ }^{5}$ (i) Antonio Mecozzi, ${ }^{6,7}$ (i) \\ Cristian Antonelli, ${ }^{6,7}$ (1) Leif K. Oxenløwe, ${ }^{1}$ and Alessandro Zavatta ${ }^{2,3}$ \\ ${ }^{1}$ CoE SPOC, DTU Fotonik, Technical University of Denmark, 2800 Kgs. Lyngby, Denmark \\ ${ }^{2}$ Istituto Nazionale di Ottica (CNR-INO), Largo E. Fermi 6, 50125 Florence, Italy \\ ${ }^{3}$ LENS and Department of Physics and Astronomy, University of Florence, 50019 Sesto Fiorentino, Italy \\ ${ }^{4}$ Department of Physics "Ettore Pancini", University of Naples "Federico II", Via Cinthia 21, 80126 Naples, Italy \\ ${ }^{5}$ Optical Communications Laboratory, Sumitomo Electric Industries, Ltd., 244-8588 Yokohama, Japan \\ ${ }^{6}$ Department of Physical and Chemical Sciences, University of L'Aquila, 67100 L'Aquila, Italy \\ ${ }^{7}$ National Laboratory of Advanced Optical Fibers for Photonics (FIBERS), CNIT, 67100 L'Aquila, Italy \\ *Corresponding author: dabac@fotonik.dtu.dk
}

Received 24 March 2021; revised 21 June 2021; accepted 9 August 2021; posted 10 August 2021 (Doc. ID 425890$) ;$ published 16 September 2021

\begin{abstract}
Multicore fibers are expected to be a game-changer in the coming decades thanks to their intrinsic properties, allowing a larger transmission bandwidth and a lower footprint in optical communications. In addition, multicore fibers have recently been explored for quantum communication, attesting to their uniqueness in transporting high-dimensional quantum states. However, investigations and experiments reported in literature have been carried out in research laboratories, typically making use of short fiber links in controlled environments. Thus, the possibility of using long-distance multicore fibers for quantum applications is still to be proven. We characterize here for the first time, to the best of our knowledge, in terms of phase stability, multiple strands of a four-core multicore fiber installed underground in the city of L'Aquila, with an overall fiber length up to about $25 \mathrm{~km}$. In this preliminary study, we investigate the possibility of using such an infrastructure to implement quantumenhanced schemes, such as high-dimensional quantum key distribution, quantum-based environmental sensors, and more, in general, quantum communication protocols. () 2021 Chinese Laser Press
\end{abstract}

https://doi.org/10.1364/PRJ.425890

\section{INTRODUCTION}

Multicore fibers (MCFs), i.e., optical fibers with multiple cores within the same cladding, are expected to be a game-changer in the next generation of telecommunication infrastructures [1-4]. In fact, MCFs present multiple advantages over standard single-mode fibers, while maintaining, at the same time, a similar performance in terms of optical loss [5,6]. Specifically, MCFs constitute a promising candidate for the implementation of space-division multiplexed transmission, and their standardization is taken into consideration. They present a lower footprint, which is of high importance in the deployment process, e.g., in telecom data-centers, where the space is limited, and they allow the use of a single amplifier for all the cores, reducing the number of resources $[7,8]$. Furthermore, MCFs represent a perfect match with photonic integrated circuits for multiple-input-multiple-output applications and are expected to be widely adopted in long-haul undersea connections [9]. Recently, MCFs have also been tested for quantum communication [10-17]. Thanks to their properties of low loss and small crosstalk between the different cores, these fibers, which are referred to as uncoupled-core MCFs, have been used for copropagating quantum and classical signals in different cores [10] or in the same core $[11,12]$ and also for transmitting high-dimensional quantum states [13-17]. High-dimensional quantum states, thanks to their intrinsic properties, allow for a higher information capacity (useful in the case of a limited photon budget or in the regime of saturating single-photon detectors) and also exhibit higher robustness to the noise affecting the quantum communication (which is critical in real-world applications) [18]. However, the transmission of highdimensional quantum states over MCFs requires phase stability between the different cores, since the quantum states are encoded in coherent superpositions of the cores of the fiber. In fact, although the improved phase stability of a single MCF, compared to a bundle of single-core fibers, was already demonstrated over $2 \mathrm{~km}$ of a seven-core uncoupled fiber (in laboratory environment), the phase stability of longer deployed MCFs has not been tested so far $[17,19,20]$. 


\section{EXPERIMENTAL SETUP}

In our experiment, we have used an optical test-bed infrastructure built in 2019 in the city of L'Aquila [4]. A single jelly-filled loose-tube cable, with an outer diameter of $6 \mathrm{~mm}$ and a total length of $6.29 \mathrm{~km}$, has been deployed in a multi-service underground tunnel with both ends accessible from the same location. The cable accommodates three different kinds of MCFs for a total of 18 strands: twelve are randomly coupled four-core MCFs (RC-4CF), four are uncoupled four-core MCFs (UC$4 \mathrm{CF}$ ), and two are uncoupled eight-core MCFs (UC-8CF). The first two kinds (RC-4CF and UC-4CF) are optimized for the C-band window $(1550 \mathrm{~nm})$, while the latter (UC-8CF) is optimized for the O-band window $(1310 \mathrm{~nm})$. All of the cores in each strand are accessible via an optical patch panel. More details are reported in the work by Hayashi and co-authors [4]. In our experiment, we focused on the UC-4CF strands. Attenuation values in the range $0.201-0.246 \mathrm{~dB} / \mathrm{km}$ have been reported in Ref. [4] for the MCF we tested. Further characterizations with respect to inter-core crosstalk [4] and time skew $[21,22]$ have also been carried out. All of the UC-4CF strands are terminated by subscriber connector (SC) MCF pigtails on both ends. This allows us to concatenate multiple strands, up to a total MCF length of $25.16 \mathrm{~km}$, or to access each core individually by connecting the strand to a fan-in fan-out device, that splits the four cores into four different single-mode fibers.

In order to adopt the MCF for quantum applications, an efficient stabilization of the relative phase between all of the different cores is required. Figure 1 shows the interferometric scheme we have implemented to test the phase stability of the UC-4CF. The output of a continuous wave laser emitting at $1550 \mathrm{~nm}$ has been injected into the common port of a $1 \times 4$ fiber beam-splitter (BS), represented by $\mathrm{BS}_{1 \times 4}$ in Fig. 1 . After the BS, we have inserted three fiber-based polarization controllers to correctly align the polarization inside the different cores of the fiber. The output ports have been connected to the four cores of a UC-4CF strand through the fan-in device. The end of this strand can be connected directly to the last part of the interferometric setup by using the fan-out kit, or to the input of another UC-4CF strand. In this way, we can adjust the overall interferometer length from $6.29 \mathrm{~km}$ to $25.16 \mathrm{~km}$ by steps of $6.29 \mathrm{~km}$. The four cores of the last connected strand, after the fan-out kit, have been injected into the input ports of two $2 \times 2$ BSs $\left(\mathrm{BS}_{1}\right.$ and $\mathrm{BS}_{2}$ in Fig. 1). Thus, two independent two-path interferometers between cores 1 and 2 and cores 3 and 4, respectively, have been realized. By monitoring one output port of each interferometer, we can measure the relative phase fluctuations between cores 1 and 2 and cores 3 and 4, as shown in Fig. 1. Moreover, by combining the remaining output ports of the two-path interferometers via a third $2 \times 2 \mathrm{BS}\left(\mathrm{BS}_{3}\right)$, we can access the relative phases between all of the cores. The three interferometer outputs were monitored with $\mathrm{p}-\mathrm{i}-\mathrm{n}$ photodiodes, resulting in three electrical signals that were collected with a National Instruments data acquisition board (NIDaq) connected to a personal computer (PC).

In order to fully and individually control the relative phases between the four uncoupled cores, a phase lock loop (PLL) has been integrated in each interferometer. The main component of each PLL is the control board (CB) composed by an ADuC7020 microcontroller unit (MCU) produced by Analog Devices. This device incorporates a five-channel 12 bit analogto-digital converter (ADC) and a four-channel 12 bit digital-toanalog converter (DAC). Thanks to the high resolution of the analog channels and to the high computational power of the MCU, this microcontroller is suitable to implement a digital proportional-integral-derivative (PID) controller with the ability to efficiently stabilize the MCF interferometers. In order to maximize the computational power available to stabilize each interferometer, we used a dedicated $\mathrm{ADuC7020}$ for each PLL. The feedback signal (FS) used by the PLL is a portion of the output of the photo-detector monitoring the same interfeometer the PLL is controlling. An ADC channel, preceded by a low-pass filter with a bandwidth of $1 \mathrm{kHz}$, is used to acquire this signal. The intensity of this signal can be related to the relative phase $\varphi$ between the cores that are involved in the two-path interferometer:

$$
\mathrm{FS}(\varphi)=\frac{M-m}{2} \cos \varphi+\frac{M+m}{2},
$$

where $M$ and $m$ are the maximum and the minimum, respectively, of the interference fringes. In order to actively control this phase, a fiber-based phase shifter has been introduced in each interferometer, as shown in Fig. 1. A DAC channel is used to control, via an high-voltage driver, the phase shifter. The firmware installed on the microcontroller can be divided into two main blocks: one generates a triangular ramp at the DAC

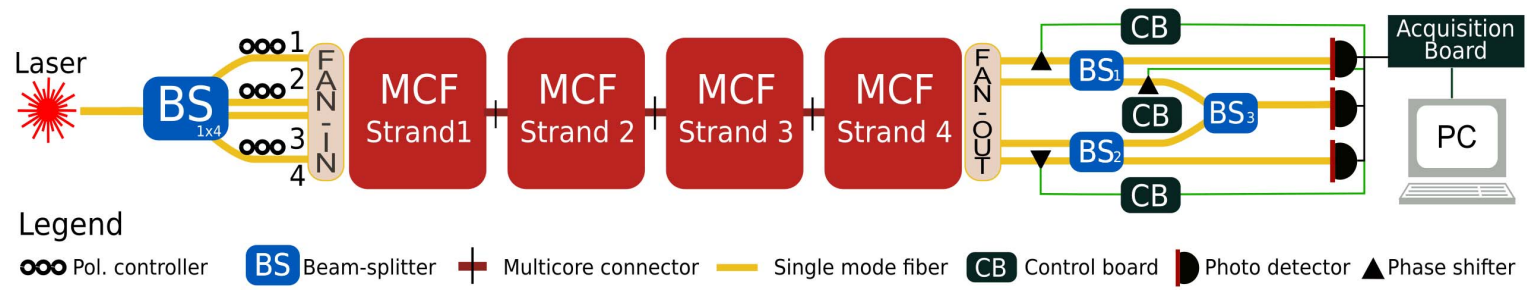

Fig. 1. Experimental setup. A continuous wave laser at $1550 \mathrm{~nm}$ is equally divided into four paths through a $1 \times 4$ beam splitter $\left(\mathrm{BS}_{1 \times 4}\right)$. Three polarization controllers are used to align the polarization inside the different cores. The four single-mode fibers are then individually connected to the fan-in input of the MCF. Thanks to the reconfigurability of the optical system, it is possible to set the number of subsequent MCF strands to be tested in the experiment (each strand is approximately $6.29 \mathrm{~km}$ in length). To connect the strands to each other, an MCF connector is used. After propagation through the $\mathrm{MCF}$, a fan-out device is used to divide the cores into four different single-mode fibers that are finally combined with each other by three $2 \times 2$ beam splitters $\left(\mathrm{BS}_{1}, \mathrm{BS}_{2}\right.$, and $\left.\mathrm{BS}_{3}\right)$. By using three automatic control boards, each driving a phase shifter, based on the measurement output of the three detectors, we can monitor and individually control the relative phase of each core. 


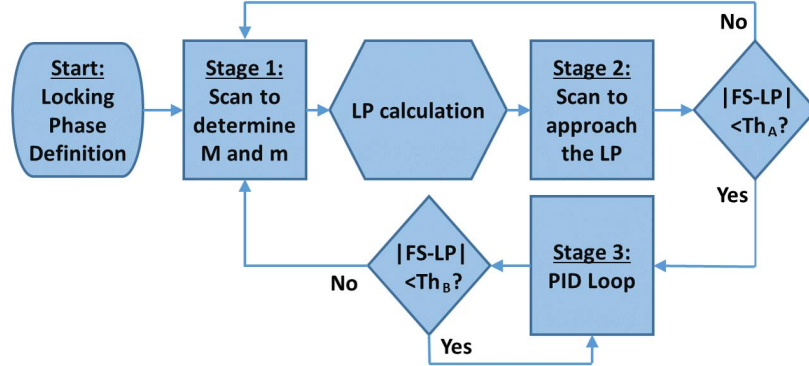

Fig. 2. Flow chart of the PID controller. This algorithm is used for controlling each phase shifter in the three different interferometers. $M$ and $m$ in stage 1 stand for the maximum and the minimum of the feedback signal (FS), respectively. LP indicates the desired locking point, while $T h_{A}$ and $T h_{B}$ are user-defined thresholds.

output to uniformly scan the interferometer relative phase, and the other contains the code that implements the digital PID used for the phase stabilization. Its behavior is described in the flowchart in Fig. 2.

The PID block is divided into three main stages. The starting point is a serial input from the user that communicates the locking phase to the microcontroller, i.e., the phase where the interferometer should be locked. This command activates the first stage, where the voltage applied to the phase shifter is scanned in order to produce a phase variation slightly bigger than $2 \pi$. Meanwhile, the FS is measured by the microcontroller ADC to find the maximum $(M)$ and the minimum $(m)$ of the interference fringes. By knowing these two parameters, it is possible to invert Eq. (1) to determine the value of the FS corresponding to the desired locking phase called the locking point (LP). The locking phase value, i.e., the LP, is a parameter that can be arbitrarily fixed by the user in the range from 0 to $2 \pi$. During the second stage, the same phase range is scanned in order to approach the LP, with a precision determined by the user-defined threshold $\mathrm{Th}_{\mathrm{A}}$. By measuring the FS during this scan, it is also possible to select the slope of the interference fringe used for locking, which allows us to stabilize the relative phase over the whole range $[0,2 \pi]$. As soon as $|F S-L P|<\mathrm{Th}_{\mathrm{A}}$, the PID loop [23] starts operating (stage 3). Otherwise, if this condition can not be reached, the algorithm restarts back from the first stage. Once activated, the PID loop keeps acting as long as the condition $|\mathrm{FS}-\mathrm{LP}|<\mathrm{Th}_{\mathrm{B}}$ remains verified. The $T h_{B}$ threshold, also defined by the user, fixes the maximum tolerable difference between FS and the desired LP, enabling us to match the locking precision to the experimental conditions. If this condition is not verified, the PID loop stops, and the algorithm starts back from the first stage, allowing for an automatic re-locking.

\section{RESULTS}

In order to phase-stabilize the cores of the MCF, we built the three interferometers as described in the previous paragraph. The first parameter to be evaluated in these interferometers is the visibility, which is directly linked to the performance of a quantum or classical communication protocol, in terms of expected error rate. In Fig. 3(a), the red (blue) curve shows the interference fringes obtained by driving, with a triangular shape, the fiber phase shifter in the two-path interferometer involving cores 1 and 2 ( 3 and 4 ) of the MCF. These two interferometers, as shown in the experimental setup in Fig. 1, are independent. We measured a visibility of $V_{1 \& 2}=$ $0.981 \pm 0.008$ and $V_{384}=0.945 \pm 0.011$. The third interferometer involves all four cores and, to evaluate its visibility, the two independent interferometers (involving cores 1 and 2 and cores 3 and 4 separately) must be locked to a specific phase in order to balance the two powers entering in $\mathrm{BS}_{3}$ of Fig. 1 . Through the first two PLLs, we have locked the two independent interferometers to around $50 \%$ of the fringe, and, exploiting the third phase shifter, we generated a triangular ramp in the overall interferometer, as shown in Fig. 3(b) with yellow color. The visibility value we measured is $V_{182 \& 3844}=$ $0.989 \pm 0.004$.

Subsequent to the visibility measurement, we investigated the possibility of stabilizing the four different cores of the MCF for a certain amount of time. The stabilization of the relative phase between the cores of an MCF is an essential property for the reliable transmission of the quantum states, both in quantum key distribution applications and in more advanced quantum network protocols. We report in Fig. 4(a) the temporal drift of the relative phases between the cores, observed with the unlocked interferometers over a continuous and free-running acquisition of $10 \mathrm{~min}$. Figure 4(b) shows the same
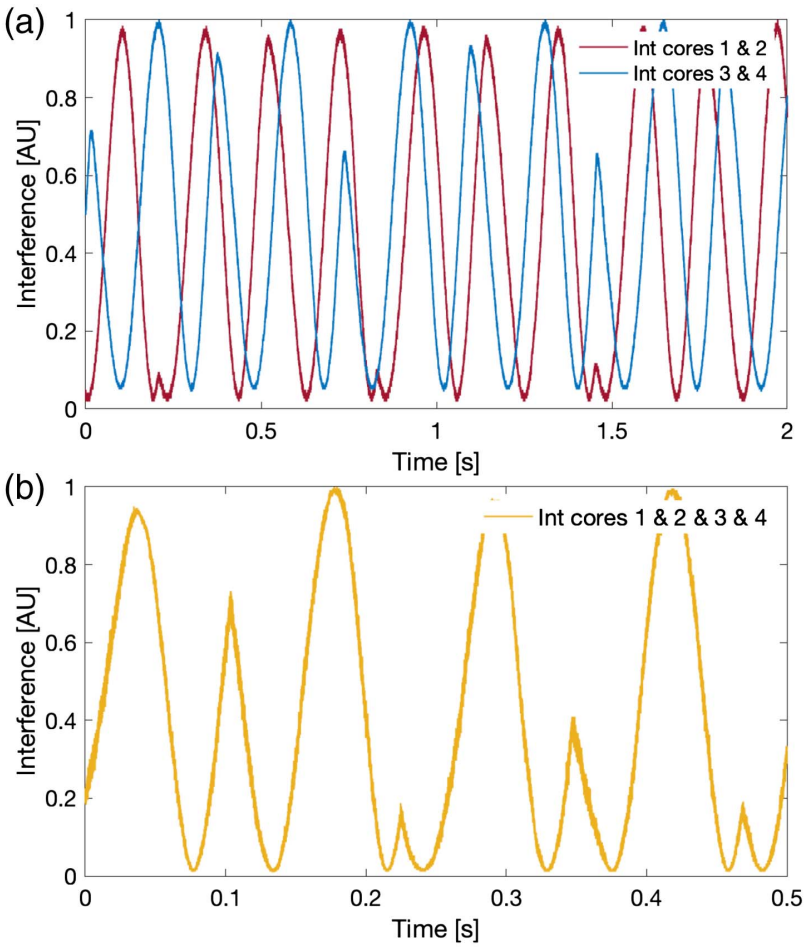

Fig. 3. Visibility fringes of the interferometers. (a) Interference signals as a function of time for the two independent interferometers between cores 1 and 2 and cores 3 and 4. Measured visibility of $V_{1 \& 2}=0.981 \pm 0.008$ and $V_{384}=0.945 \pm 0.011$. (b) Interference signal from the three interferometers. In this measurement, the first two independent interferometers are locked to a fixed position. Measured visibility of $V_{1828384}=0.989 \pm 0.004$ 

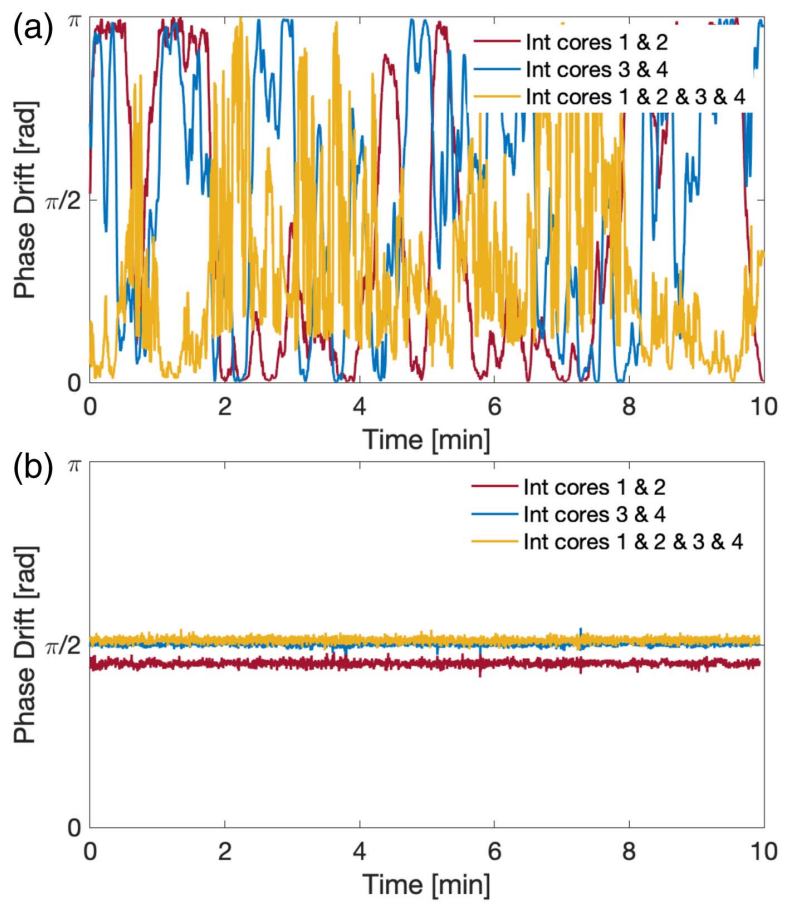

Fig. 4. Phase drifts of the non-stabilized and stabilized four concatenated strands multicore interferometers over $10 \mathrm{~min}$ acquisition. (a) Phase drift between two-core and four-core interferometers without active phase stabilization. (b) Phase drift of (a) but with active phase stabilization loops. Different colors represent the three different configurations (red and blue, two-path interferometers; yellow, four-path interferometer).

acquisition, in which we have turned on the three automatic PLL systems to actively compensate the drifts. Both figures show the behavior of the interferometer in the four-strand configuration. In order to stabilize all of the different cores, we have first locked the two independent interferometers involving cores 1 and 2 and cores 3 and 4, and, subsequently, we have locked the overall interferometer. Note that, in case of fast and abrupt drifts in the fiber, i.e., when the locking position is suddenly lost, our PLL system is able to automatically re-lock to the same position by restarting the PLL algorithm from the first stage (see Fig. 2). The whole operation, required to re-lock the three interferometers, typically takes from $3 \mathrm{~s}$ to $10 \mathrm{~s}$, which is a very short time compared to the stabilization time of $10 \mathrm{~min}$ reported in Fig. 4(b). Another important point to be highlighted is that the polarization of the different cores was stable over $100 \mathrm{~min}$ of acquisition time.

In addition, we further investigated the free-running acquisition in order to better characterize the signal phase fluctuations in the MCF for different fiber lengths. To this end, we made additional $30 \mathrm{~min}$ acquisitions, with $6 \mathrm{~Hz}$ sampling rate, of the cores' interference signals for multiple strands. In Fig. 5, we show the results of these measurements. In Fig. 5(a), we report the power spectrum of the interference signal as a function of frequency for one of the two-core interferometers (1 and 2). In Fig. 5(b), we report the same measurement for the four-core interferometer.

\section{DISCUSSION}

Optical interferometers are the basic component for optical signal processing. More specifically, fiber-based interferometers are widely used for different applications, spanning from sensing and optical communication to quantum physics and gravitational wave detections. Multipath interferometers are used for manipulating quantum states and high-dimensional unitary operations, and, in this work, we demonstrated the possibility of stabilizing a long-distance MCF through a simple and scalable setup. For example, we might imagine using a wavelength multiplexing approach for transmitting quantum and classical light in the same fiber. One of these channels could be used for stabilizing the drift of the relative phase as demonstrated in Ref. [19]. In the same paper, we also propose a full setup of a four-dimensional quantum key distribution protocol exploiting different wavelengths for stabilizing the MCF. In the same direction, we have also demonstrated that the phase drift is not directly related to the length of the MCF. In fact, by looking at Fig. 5, both configurations seem quite insensitive to the overall interferometric size. This behavior can be explained by considering that most of the phase fluctuations come from the fibers connecting the rack-mount optical patch panel to the rest of the experimental setup and to the fiber components used to implement the interferometer itself, located on a table as close to it as possible. In other words, we can assume that the most unstable portion of the interferometric apparatus is not the MCF [24].

The second evidence, from Fig. 5(b), is that the four-core interferometer is more sensitive to phase fluctuations than the
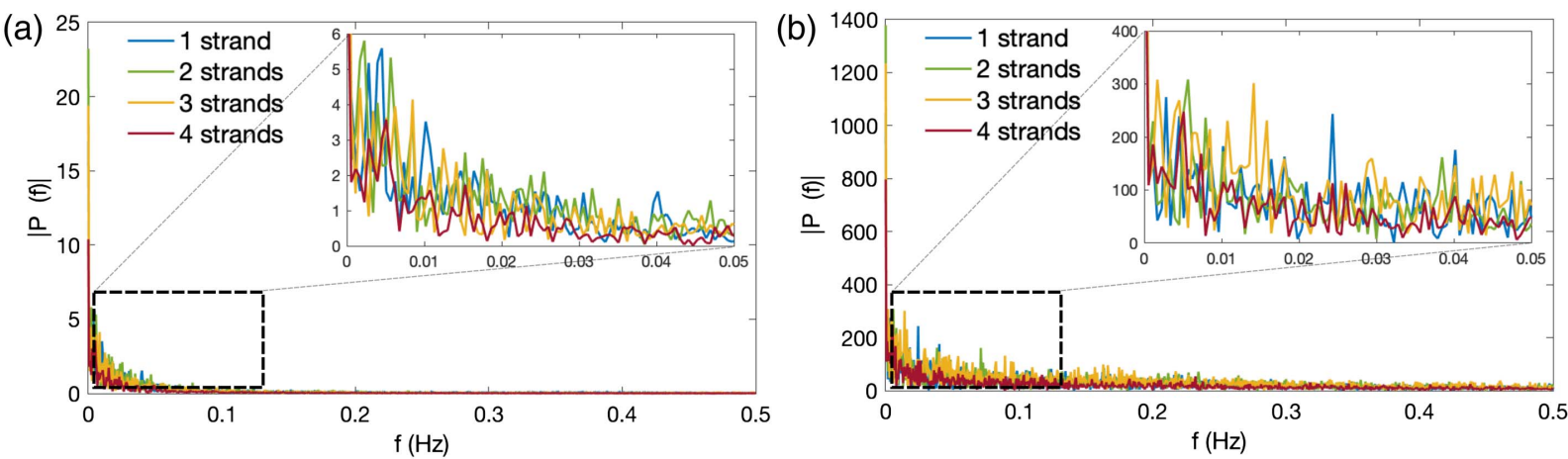

Fig. 5. Frequency analysis of the interference signals. (a) Two-core intereferometers for the four different strands. (b) Four-core interferometer for the four different strands. Different colors represent different strands. Each measurement has been acquired for 30 min in the non-locked system. 
two-path configurations. In fact, the spectral density function of the four-core interferometer is about two orders of magnitude higher compared to the two-path one. This fact, as already demonstrated in Refs. [25-29], could be quite useful for sensing applications, both classical and quantum.

Note that in our demonstration we have used cascaded interferometers for analyzing independently all of the optical signals, but new devices have been recently introduced for a multi-port BS [30]. These devices are properly designed for acting as interfaces between single-mode fibers and MCFs and could increase the overall stability of the system. In the same direction, the scalability of the entire setup for larger dimensionality (more cores) is not straightforward by using bulk and fiber optics, since multiple locking stages are necessary for stabilizing a larger amount of cores. However, it has already been demonstrated that integrated photonics could help in accomplishing this task. As an example, our previous demonstration showed the possibility of manipulating and controlling quantum states using silicon photonics [13].

Summarizing, we presented here a scalable and efficient method for stabilizing the phase drifts in an MCF. The presented method can, in principle, be applied to longer fiber distances and larger core counts by using the same technology. Our demonstration paves the way towards future investigations and applications of MCFs in quantum communication [31].

Funding. Horizon 2020 Framework Programme OpenQKD (857156), SQUARE (731473); Ministero dell'Istruzione, dell'Università e della Ricerca (INCIPIT); H2020 Science with and for Society (G5485); Danmarks Grundforskningsfond SPOC (123).

Disclosures. The authors declare that there are no conflicts of interest related to this paper.

Data Availability. Data underlying the results presented in this paper are not publicly available at this time but may be obtained from the authors upon reasonable request.

\section{REFERENCES}

1. K. Saitoh and S. Matsuo, "Multicore fiber technology," J. Lightwave Technol. 34, 55-66 (2016).

2. Y. Amma, Y. Sasaki, K. Takenaga, S. Matsuo, J. Tu, K. Saitoh, M. Koshiba, T. Morioka, and Y. Miyamoto, "High-density multicore fiber with heterogeneous core arrangement," in Optical Fiber Communications Conference and Exhibition (OFC) (IEEE, 2015), pp. 1-3.

3. H. Hu, F. Da Ros, M. Pu, F. Ye, K. Ingerslev, E. P. da Silva, M. Nooruzzaman, Y. Amma, Y. Sasaki, T. Mizuno, Y. Miyamoto, L. Ottaviano, E. Semenova, D. Zibar, M. Galili, K. Yvind, T. Morioka, and L. K. Oxenløwe, "Single-source chip-based frequency comb enabling extreme parallel data transmission," Nat. Photonics 12, 469-473 (2018).

4. T. Hayashi, T. Nagashima, T. Nakanishi, T. Morishima, R. Kawawada, A. Mecozzi, and C. Antonelli, "Field-deployed multi-core fiber testbed," in 24th OptoElectronics and Communications Conference (OECC) and 2019 International Conference on Photonics in Switching and Computing (PSC) (IEEE, 2019), pp. 1-3.

5. T. Hayashi, T. Taru, O. Shimakawa, T. Sasaki, and E. Sasaoka, "Lowcrosstalk and low-loss multi-core fiber utilizing fiber bend," in Optical
Fiber Communication Conference (Optical Society of America, 2011), paper OWJ3.

6. T. Hayashi, Y. Tamura, T. Hasegawa, and T. Taru, "Record-low spatial mode dispersion and ultra-low loss coupled multi-core fiber for ultra-long-haul transmission," J. Lightwave Technol. 35, 450-457 (2017).

7. H. Yuan, M. Furdek, A. Muhammad, A. Saljoghei, L. Wosinska, and G. Zervas, "Space-division multiplexing in data center networks: on multicore fiber solutions and crosstalk-suppressed resource allocation," J. Opt. Commun. Netw. 10, 272-288 (2018).

8. K. Abedin, T. Taunay, M. Fishteyn, D. DiGiovanni, V. Supradeepa, J. Fini, M. Yan, B. Zhu, E. Monberg, and F. Dimarcello, "Claddingpumped erbium-doped multicore fiber amplifier," Opt. Express 20, 20191-20200 (2012).

9. M. Nooruzzaman and T. Morioka, "Multi-core fibers in submarine networks for high-capacity undersea transmission systems," in Optical Fiber Communications Conference and Exhibition (OFC) (IEEE, 2017), pp. 1-3.

10. J. Dynes, S. Kindness, S.-B. Tam, A. Plews, A. Sharpe, M. Lucamarini, B. Fröhlich, Z. Yuan, R. Penty, and A. Shields, "Quantum key distribution over multicore fiber," Opt. Express 24 8081-8087 (2016).

11. D. Bacco, B. Da Lio, D. Cozzolino, F. Da Ros, X. Guo, Y. Ding, Y. Sasaki, K. Aikawa, S. Miki, H. Terai, H. Terai, T. Yashimita, J. S. Neergaard-Nielsen, M. Galili, K. Rottwitt, U. A. Andersen, T. Morioka, and L. K. Oxenløwe, "Boosting the secret key rate in a shared quantum and classical fibre communication system," Commun. Phys. 2, 140 (2019)

12. B. Da Lio, D. Bacco, D. Cozzolino, F. Da Ros, X. Guo, Y. Ding, Y. Sasaki, K. Aikawa, S. Miki, H. Terai, T. Yashimita, J. S. Neergaard-Nielsen, M. Galili, K. Rottwitt, U. A. Andersen, L. K. Oxenløwe, and T. Morioka, "Record-high secret key rate for joint classical and quantum transmission over a 37-core fiber," in IEEE Photonics Conference (IPC) (IEEE, 2018), pp. 1-2.

13. Y. Ding, D. Bacco, K. Dalgaard, X. Cai, X. Zhou, K. Rottwitt, and L. K. Oxenløwe, "High-dimensional quantum key distribution based on multicore fiber using silicon photonic integrated circuits," npj Quantum Inf. 3, 25 (2017).

14. G. Cañas, N. Vera, J. Cariñe, P. González, J. Cardenas, P. Connolly, A. Przysiezna, E. Gómez, M. Figueroa, G. Vallone, P. Villoresi, T. Ferreira da Silva, G. B. Xavier, and G. Lima, "High-dimensional decoy-state quantum key distribution over multicore telecommunication fibers," Phys. Rev. A 96, 022317 (2017).

15. D. Bacco, Y. Ding, K. Dalgaard, K. Rottwitt, and L. K. Oxenløwe, "Space division multiplexing chip-to-chip quantum key distribution," Sci. Rep. 7, 12459 (2017).

16. G. B. Xavier and G. Lima, "Quantum information processing with space-division multiplexing optical fibres," Commun. Phys. 3, 9 (2020).

17. B. Da Lio, D. Bacco, D. Cozzolino, N. Biagi, T. N. Arge, E. Larsen, K. Rottwitt, Y. Ding, A. Zavatta, and L. K. Oxenløwe, "Stable transmission of high-dimensional quantum states over a 2-km multicore fiber," IEEE J. Sel. Top. Quantum Electron. 26, 6400108 (2019).

18. D. Cozzolino, B. Da Lio, D. Bacco, and L. K. Oxenløwe, "Highdimensional quantum communication: benefits, progress, and future challenges," Adv. Quantum Technol. 2, 1900038 (2019).

19. B. Da Lio, D. Cozzolino, B. Nicola, Y. Ding, K. Rottwitt, A. Zavatta, D. Bacco, and L. K. Oxenløwe, "Path-encoded high-dimensional quantum communication over a 2 km multicore fiber," npj Quantum Inf. 7, 63 (2021).

20. A. Alarcón, J. Argillander, G. Lima, and G. B. Xavier, "Few-mode fibre technology fine-tunes losses of quantum communication systems," arXiv:2103.05018 (2021)

21. R. S. Luís, B. J. Puttnam, G. Rademacher, A. Marotta, C. Antonelli, F. Graziosi, A. Mecozzi, T. Hayashi, T. Nakanishi, S. Shinada, Y. Awaji, H. Furukawa, and N. Wada, "Evaluation of dynamic skew on spooled and deployed multicore fibers using O-band signals," in Optical Fiber Communications Conference and Exhibition (OFC) (Optical Society of America, 2020), paper T4J.4.

22. B. J. Puttnam, R. S. Luis, G. Rademacher, A. Marotta, C. Antonelli, A. Mecozzi, F. Graziosi, T. Hayashi, T. Nakanishi, Y. Awaji, H. 
Furukawa, and N. Wada, "Dynamic skew measurements in a deployed 4-core fiber," in Conference on Lasers and Electro-Optics (Optical Society of America, 2020), paper STu4R.1.

23. L. Rozsa, "Design and implementation of practical digital PID controllers," IFAC Proc. Vol. 22, 115-121 (1989).

24. X.-M. Hu, W.-B. Xing, B.-H. Liu, D.-Y. He, H. Cao, Y. Guo, C. Zhang, H. Zhang, Y.-F. Huang, C.-F. Li, and G.-C. Guo, "Efficient distribution of high-dimensional entanglement through $11 \mathrm{~km}$ fiber," Optica 7 , 738-743 (2020).

25. Z. Zhao, Z. Liu, M. Tang, S. Fu, L. Wang, N. Guo, C. Jin, H.-Y. Tam, and C. Lu, "Robust in-fiber spatial interferometer using multicore fiber for vibration detection," Opt. Express 26, 29629-29637 (2018).

26. G. M. D'Ariano and M. G. Paris, "Arbitrary precision in multipath interferometry," Phys. Rev. A 55, 2267-2271 (1997).
27. L. Gan, R. Wang, D. Liu, L. Duan, S. Liu, S. Fu, B. Li, Z. Feng, H. Wei, W. Tong, P. Shum, and M. Tang, "Spatial-division multiplexed Mach-Zehnder interferometers in heterogeneous multicore fiber for multiparameter measurement," IEEE Photon. J. 8, 7800908 (2016).

28. K. U. Schreiber and J.-P. R. Wells, "Invited review article: large ring lasers for rotation sensing," Rev. Sci. Instrum. 84, 041101 (2013).

29. G. Weihs, M. Reck, H. Weinfurter, and A. Zeilinger, "All-fiber threepath Mach-Zehnder interferometer," Opt. Lett. 21, 302-304 (1996).

30. L. Pereira, A. Rojas, G. Cañas, G. Lima, A. Delgado, and A. Cabello, "Universal multi-port interferometers with minimal optical depth," arXiv:2002.01371 (2020).

31. D. Bacco, J. F. Bulmer, M. Erhard, M. Huber, and S. Paesani, "A proposal for practical multidimensional quantum networks," arXiv:2103.09202 (2021). 